\title{
Incidencia y factores de riesgo para bacteriemia en pacientes pediátricos con quemaduras en un Centro de Atención Especializado en México
}

\author{
Teresa de J. Chávez-Velarde, Juan C. Lona-Reyes, Alejandra Riebeling-Dueñas y Luis G. Orozco-Alatorre
}

\section{Incidence and risk factors for bacteremia on pediatric patients with burns in a specialized care center in Mexico}

Background: Bacteremia events are frequent cause of mortality in burn patients and may originate from infected wounds, by bacterial translocation of endogenous microorganisms, from invasive devices or contaminated intravenous solutions. Objective: To quantify the incidence of bacteremia in pediatric patients with burns and to identify risk factors. Material and Methods: A prospective cohort study was performed in pediatric patients with burns of Hospital Civil de Guadalajara "Dr. Juan I. Menchaca". Bacteremia was diagnosed by blood cultures in patients with clinical manifestations of sepsis. Risk factors were investigated by multivariate analysis with Cox regression. Results: Were included in the cohort 260 patients, median age 3 years. The incidence of bacteremia was 9.6 events per 1,000 days patient. The most frequently isolated bacteria were Enterobacteriaceae (41.9\%), Staphylococcus aureus (22.6\%) and Pseudomonas aeruginosa (22.6\%). Factors associated with bacteremia were: burns $\geq 20 \%$ TBSA (HR 11.06; 95\% CI 4.8-25.4), deep second degree burns or higher (HR 6.9; 95\% CI 2.0-23.3) and have had two or less debridement (HR 26.4; 95\% CI 8.0-87.7). Conclusions: Patients with more extensive and deep burns with fewer debridement are at increased risk of bacteremia.

Key words: Bacteremia, burns, burn units, risk factors.

Palabras clave: Bacteriemia, quemaduras, unidad de quemados, factores de riesgo.

\section{Introducción}

$\mathrm{E}$ $174,6 \%$ de los pacientes pediátricos que sufren quemaduras tienen menos de 5 años de edad y el principal sitio de ocurrencia de los accidentes es el hogar; las causas más frecuentes son líquidos calientes en niños bajo 4 años y fuego directo en los mayores ${ }^{1,2}$. Los eventos infecciosos son una de las complicaciones más frecuentes ${ }^{2,3}$.

El tratamiento integral de los pacientes con quemaduras, que incluye reanimación hídrica, manejo ventilatorio oportuno, desbridamiento temprana de la herida, soporte nutricional y control de las infecciones, ha permitido lograr tasas de supervivencia de hasta $94,8 \%{ }^{1,4}$. En México, entre los años 1979 y 2007, la tasa de mortalidad debida a quemaduras disminuyó 69\% (desde 2,32/100 000 a $0,72 / 100000)^{5}$, siendo los pacientes más afectados aquellos que presentaron quemaduras de tercer grado (OR $1,5 \mathrm{p}=0,003) \mathrm{y} / \mathrm{o}$ con extensión mayor a $49 \% \mathrm{de}$ superficie corporal quemada (SCQ) $(\mathrm{OR} 3,3 \mathrm{p}=0,001)^{6}$.

Después del daño térmico, la superficie de las lesiones son estériles; en las siguientes horas se colonizan por microorganismos endógenos procedentes de la piel, el tracto gastrointestinal o de la vía respiratoria. En los pacientes hospitalizados la colonización puede adquirirse del ambiente hospitalario o de manos del personal de salud y puede involucrar microorganismos multi-resistentes. Staphylococcus aureus, Pseudomonas aeruginosa y especies de enterobacterias son los microorganismos más identificados ${ }^{1,4,7,8}$

Las infecciones del torrente sanguíneo pueden originarse en dispositivos invasores como catéteres venosos centrales (CVC), por translocación bacteriana del tracto digestivo o respiratorio y por administración de soluciones intravenosas contaminadas. Diez y siete por ciento de los eventos de bacteriemia son secundarios al desbridamiento quirúrgico de la quemadura; la relevancia clínica de estos eventos es incierta ${ }^{4,9}$

La presencia del síndrome de respuesta inflamatoria sistémica (SRIS) ha sido la estrategia tradicional para identificar eventos de sepsis, pero este síndrome se puede presentar hasta en $85 \%$ de los pacientes con quemaduras. The American Burn Association (ABA) sugiere establecer el diagnóstico clínico de sepsis si se presentan tres o más de las siguientes condiciones: temperatura $>39{ }^{\circ} \mathrm{C}$ o $<36,5^{\circ} \mathrm{C}$, taquicardia progresiva, taquipnea progresiva, trombocitopenia, hiperglicemia e intolerancia a la vía oral $^{1,4,10}$; la exactitud de estos criterios para el diagnós-
Hospital Civil de Guadalajara "Dr. Juan I. Menchaca". Guadalajara, Jalisco, México. División de Pediatría.

Conflictos de interés: Inexistentes Financiamiento: Institucional.

Recibido: 1 de agosto de 2016 Aceptado: 30 de marzo de 2107

Correspondencia a: Juan Carlos Lona Reyes carloslona5@hotmail.com 
tico de sepsis confirmada con hemocultivos es limitada: sensibilidad $78,2 \%$ y especificidad $49,5 \%{ }^{11}$.

El objetivo de este estudio fue cuantificar la incidencia de bacteriemia en pacientes pediátricos con quemaduras e identificar los factores de riesgo.

\section{Material y Métodos}

Se efectuó un estudio de cohorte, prospectivo, en el Hospital Civil de Guadalajara "Dr. Juan. I. Menchaca" (HCGJIM) de la ciudad de Guadalajara, Jalisco, México. La institución otorga servicios de salud a población abierta de escasos recursos económicos. La Unidad de Atención a Niños con Quemaduras cuenta con ocho habitaciones aisladas y un quirófano para el aseo y/o desbridamientos de las quemaduras y para la inserción de dispositivos intravasculares.

Se realiza reanimación hídrica a pacientes con quemaduras $\geq 10 \%$ de superficie corporal quemada (SCQ), el cálculo de los líquidos se lleva a cabo con la fórmula de Galveston, la extensión de la lesión se evalúa con la tabla de Lund-Browder y la profundidad mediante observación directa por los cirujanos plásticos. No se indican antimicrobianos sistémicos profilácticos.

Con posterioridad a la limpieza o desbridamiento de las heridas, se colocan sobre las lesiones antisépticos locales $\left(\right.$ Estericide $^{\circledR}$ ) y apósitos con plata micronizada en gel (Silvasorb ${ }^{\circledR}$ ) o en parche $\left(\right.$ Acticoat $^{\circledR}$ ). El cambio de apósitos se realiza cada tres a siete días, según las indicaciones del producto utilizado.

Período de estudio: 18 de mayo de 2014 a 12 de julio de 2016. Se incluyeron en la cohorte todos los pacientes ingresados a la Unidad de Atención a Niños con Quemaduras, excepto los procedentes de otros hospitales, que tuvieron hemocultivos positivos a su ingreso y/o que ya se conocían con diagnóstico de bacteriemia.

El cálculo del tamaño de la muestra de 260 pacientes se realizó con la finalidad de identificar factores de riesgo con Hazard Ratio $(\mathrm{HR}) \geq 2,9$, se utilizó un $\alpha$ 0,05 y $\beta$ 0,2.

\section{Definiciones}

Sepsis clínica se diagnosticó si se presentaron tres o más de las siguientes condiciones: temperatura $>39^{\circ} \mathrm{C} \mathrm{o}$ $<36,5^{\circ} \mathrm{C}$, taquicardia progresiva, taquipnea progresiva, trombocitopenia después del tercer día de la quemadura, hiperglicemia o intolerancia a la vía oral ${ }^{10}$.

En pacientes con criterio clínico de sepsis se obtuvo mediante técnica aséptica ${ }^{12}$, dos o más muestras de sangre igual o mayor a $2 \mathrm{ml}$ y se inocularon en frascos de hemocultivo (BacT/ALERT PF Pediatric FAN®). Se monitorizó el crecimiento microbiano en sistema automatizado Bact/ALERT ${ }^{\circledR 3 D}$ durante siete días. Los cultivos detectados positivos fueron resembrados en agar sangre y
McConkey. La prueba se catalogó como positiva si existió crecimiento de bacterias o levaduras en uno o más frascos, excepto si las bacterias fueron Staphylococcus coagulasa negativa, en cuyo caso se requirieron dos o más frascos con crecimiento microbiano. De las bacterias aisladas se identificó género y especie con sistema automatizado VITEK $^{\circledR} 2$.

En pacientes con CVC, uno de los hemocultivos fue obtenido a través del catéter y uno o más de venas periféricas en sitios sin heridas; el diagnóstico de bacteriemia se estableció si ambos cultivos (central y periférico) mostraron crecimiento del mismo microorganismo o si existió crecimiento de bacterias o levaduras sólo en el cultivo de vena periférica, excepto si fueron Staphylococcus coagulasa negativa (en estos eventos se requirió que ambos frascos presentaran el mismo crecimiento microbiano). En pacientes en quienes sólo la muestra obtenida de catéter fue positiva se diagnosticó colonización del catéter.

Al ingreso de cada paciente se registró la edad, género, uso de antimicrobianos profilácticos y extensión, profundidad y agente causal de la quemadura. Durante su estancia se monitorizó la utilización de dispositivos o procedimientos invasores: CVC, catéter urinario y ventilación mecánica. A su egreso se cuantificaron los días quemadura/hospitalización, días quemadura/desbridamiento y el número de desbridamientos.

\section{Análisis estadístico}

Se estimó la densidad de incidencia de bacteriemia para el total de pacientes y para subgrupos formados según la presencia o ausencia de los factores estudiados. De las variables cualitativas se estimaron frecuencias y porcentajes, la búsqueda de asociación con la variable dependiente se evaluó con riesgo relativo e intervalos de confianza al 95\% en el programa open-epi (http://www. openepi.com/Menu/OE_Menu.htm). De las variables cuantitativas se calculó mediana y rangos. La búsqueda de asociación con la variable dependiente se realizó mediante análisis bi-variado con regresión de Cox. Las variables que presentaron valor de $\mathrm{p}<0,2$ se incluyeron en análisis multivariado con regresión de Cox en programa IBM SPSS Statistics Versión 20.

El proyecto fue aprobado por los Comités de Ética e Investigación del HCGJIM con registro 1362/14.

\section{Resultados}

Se incluyeron en la cohorte 260 pacientes, mediana de edad de 3 años (mínima 0,16, máxima 15), 49,6\% (n: 129) fueron de género femenino y 50,4\% (n: 131) masculino.

La mediana de SCQ para el total de pacientes fue de $10 \%$ (mínima 1, máxima 60), en 79,2\% (n: 206) la SCQ fue menor a $20 \%$ y sólo 3,8\% (n: 10) tuvieron SCQ mayor a $40 \%$. 
Presentaron quemaduras de segundo grado superficial 43,5\% (n: 113), segundo grado profundo $42,7 \%$ (n: 111 ) y de tercer grado $13,8 \%$ (n: 36 ). Los agentes causantes de las quemaduras fueron: líquidos calientes $(70,8 \%, \mathrm{n}$ : 184), fuego (20,8\%, n: 54$)$, electricidad $(5,4 \%$, n: 14$)$ y quemadura por contacto $(3 \%, \mathrm{n}: 8)$.

Antes de su ingreso a la unidad, 9,6\% (n: 25) de los pacientes recibieron antimicrobianos profilácticos, los más utilizados (64\%) fueron cefalosporinas en monoterapia o en combinación con aminoglucósidos o vancomicina.

La mediana del tiempo entre la quemadura y el ingreso fue de un día (mínimo 0, máximo 16); 98,5\% (n: 256) se sometieron a uno o más desbridamientos quirúrgicos, la mediana de tiempo entre la quemadura y el primer desbridamiento quirúrgico fue de dos días (mínima 0 , máxima 15), la mediana de desbridamientos por paciente fue de dos intervenciones (mínima 0, máxima 14).

Se colocó CVC a 10\% (n: 26) de los pacientes, catéter urinario en $24,6 \%$ (n: 47) y conexión a ventilación mecánica en $1,9 \%$ (n: 5$)$.

Los pacientes acumularon 3.007 días de estancia hospitalaria (mediana 8, mínimo 1, máximo 108).

Treinta y cuatro por ciento (n: 89) presentó sepsis clínica, de los cuales $32,6 \%$ (n: 29) tuvieron hemocultivos positivos; la densidad de incidencia de bacteriemia fue de 9,6 eventos por 1.000 días paciente (IC 95\% 6,6-13,7).

Las bacterias aisladas fueron enterobacterias $41,9 \%$ (n: 13), Staphylococcus spp. 25,8\% (n: 8), P. aeruginosa 22,6\% (n: 7), Streptoccoccus pyogenes 6,5\% (n: 2) y Enterococcus faecalis $3,2 \%$ (n: 1 ); dos eventos de bacteriemia fueron polimicrobianos (Tabla 1$)$.

La incidencia de bacteriemia en función de variables demográficas, clínicas e intervenciones invasoras se enlistan en la Tabla 2. Para cada variable se estimó riesgo relativo (RR) e intervalos de confianza al 95\% (IC 95\%). Se observó asociación significativa de los eventos de bacteriemia con quemaduras $\geq 20 \%$ de SCQ, haber tenido dos o menos desbridamientos, la presencia de CVC y catéter urinario (Tabla 2). La extensión de la quemadura y el total de desbridamientos examinados como variables cuantitativas también fueron significativas (Tabla 3 ).

Las variables que presentaron valor de $\mathrm{p}<0,2$ se incluyeron en análisis multivariado con regresión de Cox; para obtener el modelo final se introdujo el total de las variables que cumplían el criterio y se excluyeron paso a paso, en función de su significancia estadística o la influencia que tenían para otras variables dentro del modelo. Los factores de riesgo identificados mediante análisis multivariado se enlistan en la Tabla 4.

En la cohorte sólo se registró una muerte, la cual fue secundaria a choque séptico por Klebsiella pneumoniae resistente a antimicrobianos de primera elección (cefalotina-amikacina); dos pacientes con quemaduras
Tabla 1. Microorganismos aislados de sangre en pacientes pediátricos con quemaduras

$\begin{array}{lc}\text { Especies } & \mathbf{n}(\%) \\ \text { Pseudomonas aeruginosa } & 7(22,6) \\ \text { Staphylococcus aureus } & 7(22,6) \\ \text { Enterobacter cloacae } & 4(12,9) \\ \text { Klebsiella pneumoniae } & 4(12,9) \\ \text { Klebsiella oxytoca } & 3(9,7) \\ \text { Streptococcus pyogenes } & 2(6,5) \\ \text { Enterobacter aerogenes } & 1(3,2) \\ \text { Raoultella planticola } & 1(3,2) \\ \text { Enterococcus faecalis } & 1(3,2) \\ \text { Staphylococcus epidermidis } & 1(3,2)\end{array}$

Tabla 2. Incidencia de bacteriemia en pacientes pediátricos con quemaduras en función de variables demográficas, clínicas e intervenciones invasoras

Eventos $\underset{\text { paciente }}{\text { Días }} \underset{\text { dencia* }}{\text { Inci- }}$ RR $\quad$ IC 95\% $\quad$ p

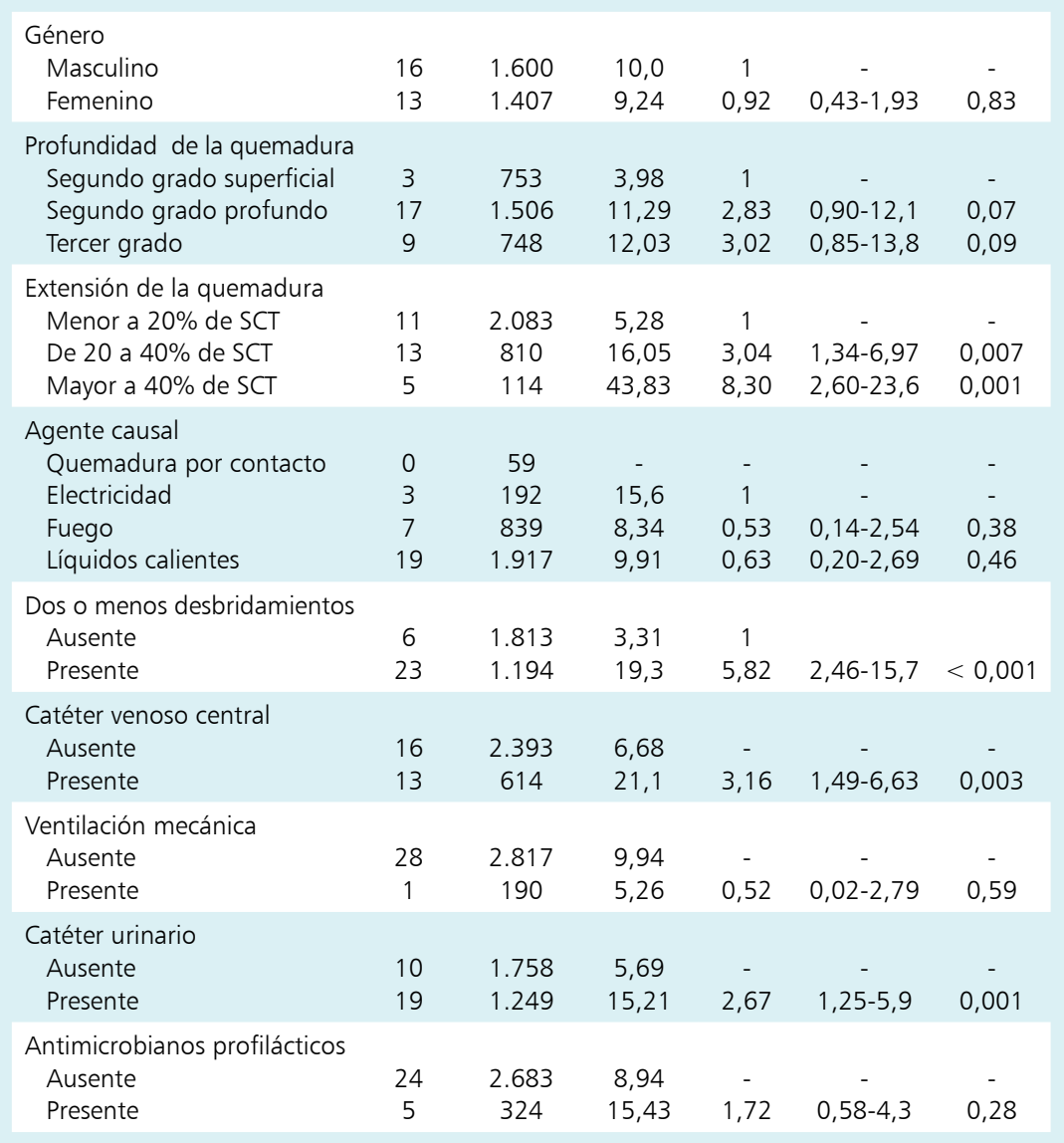

*Incidencia por 1.000 días paciente, RR: riesgo relativo, IC 95\%: intervalo de confianza al 95\%. SCT: superficie corporal total. 


\begin{tabular}{|c|c|c|c|}
\hline Variable & HR & IC $95 \%$ & $\mathbf{p}$ \\
\hline Edad & 0,99 & $0,91-1,09$ & 0,94 \\
\hline Extensión de quemadura & 1,07 & $1,04-1,09$ & $<0,001$ \\
\hline Días quemadura/hospitalización & 1,05 & $0,85-1,28$ & 0,66 \\
\hline Días quemadura/desbridamiento & 0,92 & $0,74-1,13$ & 0,42 \\
\hline Número de desbridamientos & 0,49 & $0,35-0,71$ & $<0,001$ \\
\hline
\end{tabular}

\begin{tabular}{lccc}
\multicolumn{4}{l}{ Tabla 4. Análisis multivariado (regresión de Cox) de variables asociadas a bacteriemia } \\
\hline & Hazard Ratio & IC $95 \%$ & p \\
Quemadura $\geq 20 \%$ SCT & 11,06 & $4,8-25,4$ & $<0,001$ \\
Segundo grado profundo o mayor & 6,9 & $2,0-23,3$ & 0,002 \\
\hline Dos o menos desbridamientos & 26,4 & $8,0-87,7$ & $<0,001$
\end{tabular}

$>40 \%$ de SCQ fueron trasladados a Shriner's Hospitals for Children-Galveston.

\section{Discusión}

De manera similar a lo reportado en diferentes estudios, la principal causa de quemaduras en niños atendidos en HCGJIM fueron líquidos calientes $(70,8 \%)$ y fuego $(20,8 \%)^{1,2,7}$. La incidencia de bacteriemia fue de 9,6 eventos por 1.000 días paciente, y las bacterias más identificadas fueron enterobacterias, $P$. aeruginosa y $S$. aureus.

Se ha propuesto que los eventos de bacteriemia que se presentan después del desbridamiento quirúrgico de las heridas pueden ser transitorios y es incierta su trascendencia clínica ${ }^{9}$. En nuestra cohorte, seis eventos de bacteriemia se presentaron en las $72 \mathrm{~h}$ posteriores al primer desbridamiento; todos los pacientes recibieron tratamiento antimicrobiano debido a la presencia de sepsis clínica. Ramos GE y cols. ${ }^{9}$, demostraron mediante cultivos de sangre y secreción de herida simultáneos, que $17 \%$ de los procedimientos de desbridamiento se asocian a bacteriemia, con tendencia a ser más frecuentes en quemaduras mayores (RR 3,78; IC 95\% 0,93-15,3). Otras fuentes de infecciones del torrente sanguíneo son las manos de personal médico, la colonización de catéteres y la translocación bacteriana favorecida por hipoperfusión intestinal ${ }^{7}$.

Devrim I y cols. ${ }^{13}$, observaron diferencia en la etiología de bacteriemia en niños con quemaduras en función del tiempo posterior al daño térmico; en la primera semana,
90\% de los eventos fueron causados por cocáceas grampositivas, mientras después de la segunda semana las bacterias prevalentes fueron bacilos gramnegativos (42,8\%). En este estudio, la mediana de tiempo de presentación de bacteriemia fue de 7 días (mínimo 2, máxima 31) y no se identificó diferencia en la etiología según el tiempo posterior a la quemadura (p 0,39).

En los microorganismos aislados en el presente estudio fue infrecuente la resistencia a antimicrobianos de primera elección, $85,7 \%$ de los aislados de $S$. aureus fueron sensibles a meticilina, sólo una cepa de $P$. aeruginosa fue multi-resistente y $92,3 \%$ de las enterobacterias fueron sensibles a amikacina, piperacilina/tazobactam, cefalosporinas y meropenem. En semejante forma a lo observado en nuestro estudio, Hassen y cols. ${ }^{7}$, identificaron que las bacterias prevalentes en infecciones nosocomiales en niños con quemaduras fueron $S$. aureus $(30,4 \%), P$. aeruginosa (23,8\%) y Enterobacter cloacae (16\%), y más de $88 \%$ de estas cepas fueron sensibles a antibacterianos de primera línea.

En nuestro estudio se observó que la extensión de la quemadura $\geq 20 \%$ de SCQ incrementa diez veces el riesgo de bacteriemia, y las quemaduras de segundo grado profundo y/o de tercer grado incrementan el riesgo 5,9 veces. Es probable que la extensión y la profundidad de la quemadura sean proporcionales a la respuesta inmunológica que, en una fase temprana, se caracteriza por un estado pro inflamatorio (incremento de interleukina 1 , interleukina 6 , factor de necrosis tumoral e interferón gamma) seguido de una fase antiinflamatoria y activación permanente del sistema del complemento, con pobre capacidad fagocítica y de adherencia de macrófagos y neutrófilos que en conjunto promueven un estado de inmunosupresión ${ }^{1}$.

Rodgers GL y cols. ${ }^{3}$, en una observación similar a nuestros resultados, advirtieron que la lesión por inhalación $(\mathrm{p}<0,0001)$, las quemaduras $>30 \%$ SCQ $(\mathrm{p}<0,0001)$ y lesiones de espesor total $(\mathrm{p}<0,0001)$ incrementan el riesgo de infección en niños con quemaduras. Hassen y cols. ${ }^{7}$, indicaron que las consecuencias de las quemaduras están relacionadas con su extensión, y que las lesiones $>20 \%$ de SCQ se asocian con mayor riesgo de infección y de muerte. Vyles D y cols. ${ }^{14}$, estimaron que la presencia de CVC (p 0,03) y las quemaduras de segundo (p 0,03) y tercer grado $(\mathrm{p} 0,04)$ son predictores de infección bacteriana en niños febriles con quemaduras.

Estudios previos han identificado que el desbridamiento temprano de la quemadura elimina el estímulo inflamatorio y modifica el riesgo de $\operatorname{sepsis}^{1,15}$. En esta investigación, el promedio de tiempo entre la quemadura y el primer desbridamiento no fue diferente entre pacientes con y sin bacteriemia (2,2 días vs 2,5 días, p 0,55); sin embargo, en el análisis multivariado se observó que los pacientes con quemaduras $>20 \%$ de SCQ y de segundo 
grado profundo o mayores, que tuvieron dos o menos desbridamientos, presentaron mayor probabilidad de bacteriemia.

En el presente estudio, 9,6\% (n: 25) de los pacientes recibieron antimicrobianos profilácticos por vía sistémica antes de ingresar a la cohorte; sin embargo, de manera semejante a lo reportado por Barajas-Nava y cols. ${ }^{16}$, no se cuantificó diferencia en la ocurrencia de bacteriemia en pacientes con y sin antimicrobianos. Rosanova y cols. ${ }^{17}$, observaron que el uso de antimicrobianos profilácticos en niños con quemaduras incrementa el riesgo de infecciones (RR 5,22; IC 95\% 1,26-21,63).

La baja mortalidad $(0,38 \%)$ cuantificada en este estudio es probable que se deba a que la mayoría de pacientes de la cohorte presentaron quemaduras con menos de $20 \%$ de SCQ o de segundo grado superficial; Cuenca-Pardo y cols. $^{2}$, en estudio realizado en Magdalena de las Salinas, México, reportaron una mortalidad mayor de $0,87 \%$.

Las limitaciones de este estudio son un tamaño de muestra insuficiente para subgrupos de pacientes con quemaduras de tercer grado o de extensión mayor a $40 \%$. Debido a que no se contó con cultivos cuantitativos de piel, no fue posible establecer asociación de los eventos de bacteriemia con infección probada de la herida; los diagnósticos de infección de la herida (n: 4) se realizaron mediante observación y sólo en un caso existió infección concomitante de piel y el torrente sanguíneo.

\section{Conclusiones}

La incidencia de bacteriemia fue de 9,6 eventos por 1.000 días paciente. Las bacterias más frecuentes aisladas fueron enterobacterias $(41,9 \%), S$. aureus $(22,6 \%)$ y
P. aeruginosa $(22,6 \%)$. Los pacientes con quemaduras $\geq 20 \%$ SCQ (HR 11,06; IC 95\% 4,8-25,4) y de segundo grado profundo o mayores (HR 6,9; IC 95\% 2,0-23,3) con dos o menos desbridamientos (HR 26,4; IC 95\% 8,0-87,7) presentan mayor riesgo de bacteriemia.

\section{Resumen}

Introducción: Los eventos de bacteriemia son causa frecuente de mortalidad en pacientes con quemaduras y pueden originarse en heridas infectadas, por translocación bacteriana de microorganismos endógenos, por dispositivos invasores o por soluciones intravenosas contaminadas. Objetivo: Cuantificar la incidencia de bacteriemia en pacientes pediátricos con quemaduras e identificar los factores de riesgo. Material y Métodos: Estudio de cohorte prospectivo, en población pediátrica con quemaduras del Hospital Civil de Guadalajara "Dr. Juan I. Menchaca". En pacientes con manifestaciones clínicas de sepsis se diagnosticó bacteriemia mediante cultivos de sangre. Se indagaron factores de riesgo con análisis multivariado con regresión de Cox. Resultados: Se incluyeron en la cohorte 260 pacientes, mediana de edad 3 años. La incidencia de bacteriemia fue de 9,6 eventos por 1.000 días paciente. Las bacterias más frecuentes aisladas fueron enterobacterias (41,9\%), Staphylococcus aureus $(22,6 \%)$ y Pseudomonas aeruginosa $(22,6 \%)$. Los factores asociados a bacteriemia fueron: quemaduras $\geq 20 \%$ de superficie corporal quemada (HR 11,06; IC 95\% 4,8-25,4), quemaduras de segundo grado profundo o mayores (HR 6,9; IC 95\% 2,0-23,3) y haber tenido dos o menos desbridamientos (HR 26,4; IC 95\% 8,0-87,7). Conclusiones: Pacientes con quemaduras más extensas y profundas, con menor número de desbridamientos presentan mayor riesgo de bacteriemia.

\section{Referencias bibliográfícas}

1.- Church D L, Slaba I, Winston B W, Lindsay. Burn Infections. En: Rosenbert E, De Long EF, Lory S, Stackebrandt E, Thompson F, Editores. The Prokaryotes. Berlin Heidelberg: SpringerVerlag; 2013. p. 353-74.

2.- Cuenca-Pardo J, Álvarez-Díaz C J, ComprésPichardo T A. Related factors in burn children. Epidemilogical study of the Burn Unit at the "Magdalena de las Salinas" Traumatology Hospital. J Burn Care Res 2008; 29 (3): 468-74.

3.- Rodgers G L, Mortensen J, Fisher MC, Lo A, Cresswell A, Long SS. Predictors of infectious complications after burn injuries in children Pediatr Infect Dis J 2000; 19 (10): 990-5.

4.- Murray C K. Burns. En: Bennett J, Dolin R, Blaser MJ, Eds. Mandell, Douglas, and
Bennett's Principles and Practice of Infectious Diseases. 8 ed. New York: Elsevier Saunders; 2015. p. 3505-9.

5.- Orozco-Valerio M J, Miranda-Altamirano R A, Méndez Magaña A C, Celis A. Tendencia de mortalidad por quemaduras en México, 19792009. Gac Med Mex 2012; 148: 349-57.

6.- Ibarra-Estrada M A, Chávez-Peña Q, GarcíaGuardado D I, López-Pulgarín J A, Aguirre-

Ávalos G, Corona-Jiménez. A 10-year experience with major burns from a non-burn intensive care unit. Burns 2014; 40 (6): 122531.

7.- Hassen A F, Khalifa S B, Daiki M. Epidemiological and bacteriological profiles in children with burns. Burns 2014; 40: 1040-5.

8.- Öncül O, Öksüz S, Acar A, Ülkür E, Turhan $\mathrm{V}$, Uygur F, et al. Nosocomial infection characteristics in a burn intensive care unit:
Analysis of an eleven-year active surveillance. Burns 2014; 40 (5): 835-41.

9.- Ramos G E, Resta M, Durlach R, Patiño O, Bolgiani A, Prezzavento G, et al. Peri-operative bacteraemia in burn patients. What does it mean? Ann Burns Fire Disasters 2006; 19 (3): 130-5.

10.- Greenhalgh D G, Saffle J R, Holmes J H, Gamelli R L, MD, Palmieri T L, Horton J W, et al. American Burn Association Consensus Conference to Define Sepsis and Infection in Burns. J Burn Care Res 2007; 28 (6): 776-90.

11.- Hogan B K, Wolf S E, Hospenthal D R, D'Avignon L C, Chung K K, Yun H C, et al. Correlation of American Burn Association sepsis criteria with the presence of bacteremia in burned patients admitted to the intensive care unit. J Burn Care Res 2012; 33 (3): 371-8. 
12.- Hall K K, Lyman J A. Updated review of blood culture contamination. Clin Microbiol Rev 2006; 19 (4): 788-802.

13.- Devrim İ, Kara A, Düzgöl M, Karkıner A, Bayram N, Temir G, et al. Burn-associated bloodstream infections in pediatric burn patients: Time distribution of etiologic agents. Burns 2017; 43: 144-8.
14.- Vyles D, Sinha M, Rosenberg D I, Foster K N, Tran M, Drachman D. Predictors of serious bacterial infections in pediatric burn patients with fever. J Burn Care Res 2014; 35: 291-5.

15.- Sheridan R L. Burn Care. Results of technical and organizational progress. JAMA 2003; 290 (6): 719-22.

16.- Barajas-Nava L A, López-Alcalde J, Roqué i
Figuls M, Solà I, Bonfill Cosp X. Antibiotic prophylaxis for preventing burn wound infection. Cochrane Database Syst Rev 2013 6; 6: CD008738.

17.- Rosanova M T, Stamboulian D, Lede R. Infecciones en los niños quemados: análisis epidemiológico y de los factores de riesgo. Arch Argent Pediatr 2013; 111 (4): 303-8. 